\title{
Verbal communication is preferred to coordinate neurological services in a primary care setting
}

\author{
Hillary L Woodworth ${ }^{1}$, Muhammad U Farooq ${ }^{1,2}$ and Philip B Gorelick ${ }^{1,2}$ \\ ${ }^{1}$ Michigan State University College of Human Medicine, Grand Rapids, MI, USA \\ ${ }^{2}$ Hauenstein Neurosciences, Mercy Health Saint Mary's Hospital, Grand Rapids, MI, USA
}

\begin{abstract}
Background: Poor communication between healthcare providers leads to medical errors, which cost an estimated $\$ 19.5$ billion annually in the United States and lead to adverse patient outcomes. One model of healthcare delivery that aims to foster collaboration amongst providers is the medical home, whereby patients receive integrated care in a single setting with primary care and consultants working in close collaboration. Even in integrated healthcare systems with uniform electronic medical record (EMR) systems, providers face challenges in coordinating primary care with specialty care including neurology.
\end{abstract}

Objective: To investigate how primary care team members prefer to communicate with specialty offices to coordinate care of mutual patients. Primary care team members included physicians, advanced practice professionals (APPs), and office staff in the greater Grand Rapids, Michigan area.

Methods: A four-item, multiple choice questionnaire was administered to primary care team members. Participants were asked what modality was preferred to coordinate both direct (e.g. treatment plan, diagnostic work-up) and indirect (e.g. scheduling, billing, insurance) patient care matters with specialty practices. We also queried whether primary care team members would be amenable to three different interventions aimed at improving management of patients with neurological conditions in a primary care setting.

Results: 174 primary care team members participated in the survey. Communication in person or by telephone are significantly preferred for coordination of both direct and indirect patient care matters $(p<0.0001)$ but did not vary significantly by team member role. Additional preferred modalities include fax, electronic medical record chat (EMR chat), and Doc Halo pager. To improve management of outpatients with neurological conditions, primary care physicians and APPs expressed support for monthly neurologist visits (e.g., "lunch and learn" sessions), neurology telemedicine services, or a complaint-focused order set in the EMR.

Conclusions: These data suggest that person-to-person interaction is preferred by primary care providers and staff for coordination of specialty care. In sum, these findings support interventions utilizing person-to-person collaboration to improve management of patients across both primary and specialty care.

\section{Introduction}

Poor communication between healthcare providers leads to medical errors, which cost an estimated $\$ 19.5$ billion annually in the United States and lead to adverse patient outcomes [1-4]. One model of healthcare delivery that aims to foster collaboration amongst providers is the medical home, whereby patients receive integrated care in a single setting with primary care and consultants working in close collaboration [5]. With the passage of the Patient Protection and Affordable Care Act in 2010, medical providers were incentivized to improve patient outcomes through increased collaboration [6,7]. However, even in integrated healthcare systems with uniform electronic medical record (EMR) systems, providers face challenges in coordinating primary care with specialty care including neurology [8]

Patient-physician communication is at the center of the clinical experience [9]. Unfortunately, the modern explosion of technology has eroded the 'old style doctor-patient relationship' as the patient may be viewed as a list of radiological imaging and other tests results [10]. Better physician communication skills may improve patient outcomes and satisfaction $[11,12]$. The principles of good communication can be taught and learned. Although beyond the scope of this article, the reader is referred to other reviews on the topic of physician communication $[11,12]$. The same communication principles may apply to physicianto-staff and staff-to-staff communication in an office setting.
In the United States, neurologists comprise $1.5 \%$ of physicians and see approximately 15 million office visits annually $[13,14]$. Previous work has shown that interventions aimed at improving collaboration between primary care and specialists leads to better patient outcomes [15-22], however, these studies have focused on medical specialties outside of neurology. Thus, it is unknown how increased communication between primary care and neurology modulates patient outcomes. One report found that primary care providers perceived neurology as one of the most difficult specialties with whom to communicate [8], underscoring the need to better understand how to promote collaboration between primary care and neurology. To address this question, we surveyed primary care providers and office staff to understand what modalities are preferred to communicate with specialty offices across a variety of patient care matters.

${ }^{\star}$ Correspondence to: Philip B Gorelick, Mercy Health Hauenstein Neurosciences, 220 Cherry Street SE, Grand Rapids, MI 49503, USA, Tel: 616-685-5050, E-mail: pgorelick@thorek.org

Key words: neurology, primary care, specialists, communication preference

Received: March 18, 2020; Accepted: April 08, 2020; Published: April 13, 2020 


\section{Methods}

\section{Site visits and participants}

Mercy Health Physician Partners (MHPP) has 20 outpatient primary care sites in the greater Grand Rapids, Michigan region and register over 283,000 patient visits annually. Visits to the sites were arranged between May and July 2016 to learn how primary care offices preferred to communicate with the hub neurological specialty office on indirect (e.g., scheduling a specialty office visit) and direct (e.g., management and diagnosis) patient care issues. The primary care offices included both family medicine and internal medicine practices. Since January 2016, MHPP outpatient offices have used the Athenahealth electronic medical record (EMR) system (Watertown, MA). Based on a convenience sample, visits were completed at 15 of the primary care offices (75\%) according to scheduling availability.

All primary care team members and office staff were invited to attend group sessions led by one of the authors (PBG) wherein approximately 45 minutes were spent explaining the purpose of the visit, defining operational definitions of terms used on a survey instrument designed to learn about preferences for communication with a specialty neurology office hub, and for administering the survey. As part of the survey, participants were asked to identify their role within the primary care team. Respondents identifying themselves as resident physicians were included in the "physician" category. "Advanced practice professional" (APP) included both physician assistants and nurse practitioners. "Other" included participants whose role did not fall under the category of physician, APP, medical assistant (MA), or nurse. The study was approved by the Mercy Health Regional Institutional Review Board.

\section{Survey description, analysis and goals}

Paper copies of the four-item survey were administered in-person at each site visit by PBG, and completed questionnaires were collected the same day. The only identifying information on each survey was the office location. No surveys were found to have additional personal information that may have compromised the anonymity of the participants. Each completed survey was assigned a unique number and results were manually entered into an electronic database. To further de-identify the data prior to analysis, office location was removed from each set of survey results. Data were analysed in SPSS 25 (IBM) using chi-square tests with post-hoc Bonferroni corrections for multiple comparisons where appropriate to assess whether responses varied by job title.

The two major goals of the survey were to determine how primary care team members prefer to communicate with 1) specialty offices regarding indirect patient care activities (e.g., billing, scheduling, insurance coverage) and 2) specialty providers regarding direct patient care activities (e.g., treatment plan, recommended diagnostic tests and medications). Primary care offices in the MHPP utilize a team-based approach whereby all team members including providers and office staff participate in both direct and indirect patient matters, thus all team members were asked to report their preferences for both types of matters. For example, primary care office staff may be asked to contact a specialist provider to connect them directly to the primary care provider. Participants were provided with six different communication modalities to choose from and were allowed to choose more than one modality. With regard to these choices, "person-to-person" means direct, verbal exchange of information either face-to-face or by telephone. "EMR chat" refers to instant messaging within the EMR, and "fax" refers to a facsimile transmission. A secure paging system is used within MHPP (Halo Communications, Cincinnati, OH), an analog secure text messaging system operated by providers on their personal cell phones. "Video conference" was analysed as a separate category and was not considered to be a form of person-to-person communication for the purposes of the study.

As a separate survey question of the four-question survey, physicians and APPs were asked how to best improve management of common conditions within the primary care setting that may eventually require neurology consultation. An example scenario given to participants was a patient with recurrent headaches, a common medical challenge in primary care clinics that may require input from a neurologist for successful management. However, primary care providers often have difficulty discerning which patients would benefit from a specialty referral to a neurologist. With regard to the response options, "physician visit" meant a monthly visit to the primary care clinic by a physician specialist (in this case a neurologist to discuss challenging cases at for example, a "lunch and learn" session). "Telemedicine service" referred to having a specialty provider on call during office hours to answer questions from primary care providers who have a challenging case or other questions. "Order set in EMR" meant having an order set (i.e., care pathway) designed by a specialist for workup of a challenging case by a primary care provider and to also help to determine if a referral to a specialist might be needed.

\section{Results}

Primary care team members and office staff were first asked to identify their occupational roles (Table 1). A total of 174 surveys were included in the analysis of the 15 primary care offices, with a majority of staff physicians (33\%) and 2 of 58 self-identifying as resident physicians. The 26 participants identifying as "other" included 15 administrators, 6 referral coordinators, 2 health professional students, 1 behavioural health specialist, 1 phlebotomist, and 1 care manager.

The methods by which primary care team members prefer to communicate with specialty offices regarding non-patient care or indirect activities (i.e. scheduling, billing etc.) are shown in table 2. The most frequently preferred method was person-to-person contact (44\% of total responses) followed by EMR chat (25\%) and fax (18\%). An initial chi-square test showed a significant difference amongst the proportions of responses $(p<0.0001)$, indicating that preferences were significantly different from expected proportions due to chance. We asked whether responses varied by provider role and found that role did not predict preference for person-to-person communication, EMR chat or fax using additional chi-square testing with Bonferroni correction for multiple comparisons $(p=0.23$ to 0.84 ). Interestingly however, physicians were more likely to prefer using a secure pager to communicate regarding indirect patient care activities compared to other primary care team members $(p<0.0016)$. Options including email and video conferencing were not frequently selected, however

Table 1. Self-identified job roles of primary care office participants

\begin{tabular}{|c|c|}
\hline Role & n (\% total) \\
\hline Physician & $58(33 \%)$ \\
\hline MA & $32(18 \%)$ \\
\hline Nurse & $32(18 \%)$ \\
\hline APP & $26(15 \%)$ \\
\hline Other & $26(15 \%)$ \\
\hline Total & 174 \\
\hline
\end{tabular}

APP: Advanced practice professional and includes nurse practitioners and physician assistants; MA: Medical assistant 
team members self-identifying in the "other" category were more likely to choose video conferencing $(p<0.0016)$.

Participants were next asked how they prefer to communicate with specialty providers regarding matters of direct patient care (i.e. treatment plan, symptoms, diagnostic workup), and results are shown in table 3. Similar to communication preferences regarding indirect patient care, primary care team members preferred person-to-person communication (54\%), followed by EMR chat (24\%) and a secure pager (12\%). An initial chi-square test revealed that preferences were statistically significant $(p<0.0001)$. Job role did not significantly predict preference for person-to-person communication; however, physicians were again more likely to prefer a secure pager compared to other team members $(p<0.0016)$. EMR chat, a secure pager, and fax were the next most frequently chosen responses, while email and video conferencing were rarely selected. Taken together, these data suggest that in an integrated healthcare system, person-to-person communication was most preferred for communication pertaining to both direct and indirect patient care activities, and this preference did not vary substantially across a diverse range of job roles in the outpatient primary care setting.
Next, we asked whether preference varied by type of communication (i.e. direct patient care with specialty providers vs. indirect patient care with specialty office staff). To address this, we combined the data shown in tables 2 and 3 and assessed via chi-square whether the proportions of responses differed significantly for indirect compared to direct patient care matters. As anticipated, the preference for person-to-person communication did not vary by type of patient care issue, nor did other communication modalities with the exception of fax, which was significantly preferred for indirect matters compared to direct matters $(p<0.01)$ (Figure 1).

Finally, given that primary care offices are often the gateway for referral to specialty care, we assessed interest amongst physicians and APPs in implementation of different modalities aimed at improving knowledge and management of common conditions dealt with in specialty practices before and after the referral process. In the survey, neurology was used as the specialty of interest, and physicians and APPs were asked whether they would prefer monthly visits by a neurologist to the primary care site to discuss cases, a telemedicine service staffed by a neurologist available by phone during business hours, a concernspecific order set in the EMR (e.g., a headache care management

Table 2. Preferences for communication with specialty offices regarding indirect patient care matters

\begin{tabular}{|c|c|c|c|c|c|c|}
\hline Response & n (\% total) ${ }^{a}$ & Physician (\%) & MA (\%) & Nurse (\%) & APP (\%) & Other $(\%)$ \\
\hline Person-to-Person & $107(44 \%)$ & $29(39 \%)$ & $25(53 \%)$ & $22(48 \%)$ & $15(43 \%)$ & $16(43 \%)$ \\
\hline Fax & $44(18 \%)$ & $10(13 \%)$ & $12(26 \%)$ & $10(22 \%)$ & $7(20 \%)$ & $5(14 \%)$ \\
\hline EMR Chat & $60(25 \%)$ & $20(27 \%)$ & $9(19 \%)$ & $9(20 \%)$ & $13(37 \%)$ & $9(24 \%)$ \\
\hline Secure Pager & $19(8 \%)$ & $13(17 \%)^{b}$ & $1(2 \%)$ & $3(7 \%)$ & 0 & $2(5 \%)$ \\
\hline Email & $9(4 \%)$ & $3(4 \%)$ & 0 & $2(4 \%)$ & 0 & $3(8 \%)$ \\
\hline Video Conference & $9(4 \%)$ & 0 & 0 & 0 & 0 & $2(5 \%)^{c}$ \\
\hline Total & 241 & 75 & 47 & 46 & 35 & 37 \\
\hline
\end{tabular}

${ }^{\text {a }}$ Proportions of responses were significantly different by chi-square $(p<0.0001)$

${ }^{\mathrm{b}}$ Physicians were more likely to choose a secure pager as a communication mode $(p<0.0016)$

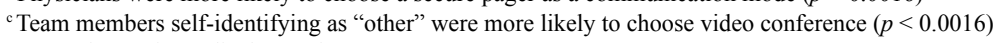

EMR: Electronic medical record

Table 3. Preferences for communication with specialty providers regarding direct patient care matters

\begin{tabular}{|c|c|c|c|c|c|c|}
\hline Response & n (\%total) ${ }^{a}$ & Physician & MA & Nurse & APP & Other \\
\hline Person-to-Person & $140(54 \%)$ & $45(51 \%)$ & $26(55 \%)$ & $25(56 \%)$ & $21(54 \%)$ & $23(53 \%)$ \\
\hline Fax & $18(7 \%)$ & $3(3 \%)$ & $4(9 \%)$ & $8(18 \%)$ & $1(3 \%)$ & $2(5 \%)$ \\
\hline EMR Chat & $61(24 \%)$ & $18(20 \%)$ & $13(28 \%)$ & $8(18 \%)$ & $14(36 \%)$ & $8(21 \%)$ \\
\hline Secure Pager & $31(12 \%)$ & $19(22 \%)^{b}$ & $2(4 \%)$ & $4(9 \%)$ & $3(8 \%)$ & $3(8 \%)$ \\
\hline Email & $5(2 \%)$ & $2(2 \%)$ & $1(2 \%)$ & 0 & 0 & $2(5 \%)$ \\
\hline Video Conference & $3(1 \%)$ & $1(1 \%)$ & $1(2 \%)$ & 0 & 0 & $1(3 \%)$ \\
\hline Total & 258 & 88 & 47 & 45 & 39 & 39 \\
\hline
\end{tabular}

a Proportions of responses were significantly different by chi-square $(p<0.0001)$

${ }^{\mathrm{b}}$ Physicians were more likely to choose a secure pager as a preferred communication mode $(p<0.0016)$

EMR: Electronic medical record

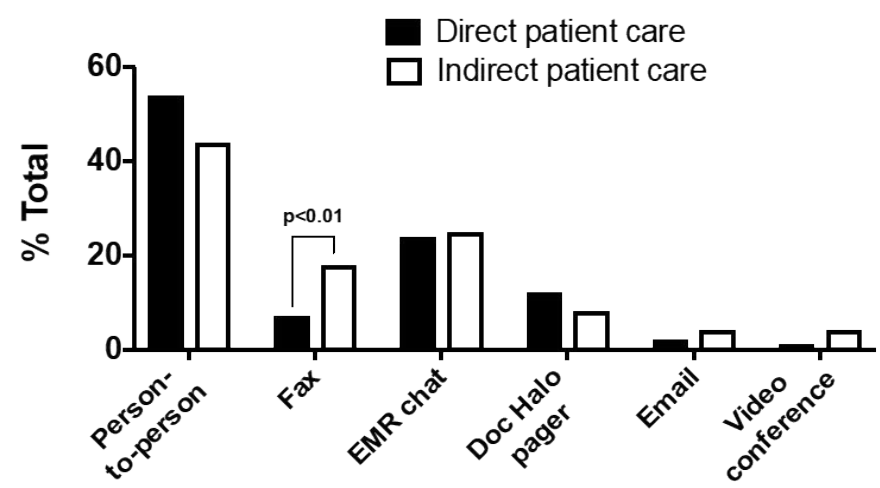

Figure 1. Comparison of communication mode preferences by type of patient care matter

The percentages of total responses for each communication mode are displayed for both communication with specialty offices (indirect patient care) and specialty providers (direct patient care) and compared to each other by chi-square testing with Bonferroni post-tests ( $p<0.01$ is significant). EMR: Electronic medical record 
Table 4. Survey of physicians and APPs regarding implementation of strategies to improve management of conditions commonly requiring specialty referral

\begin{tabular}{|c|c|c|c|}
\hline Response Options & n (\%total) & Physician (\%) & APP (\%) \\
\hline Physician Visit & $35(26 \%)$ & $22(25 \%)$ & $13(30 \%)$ \\
\hline Telemedicine Service & $56(42 \%)$ & $41(46 \%)$ & $15(34 \%)$ \\
\hline Order Set in EMR & $42(32 \%)$ & $26(29 \%)$ & $16(36 \%)$ \\
\hline No Change & 0 & 0 & 0 \\
\hline Total & 133 & 89 & 44 \\
\hline
\end{tabular}

EMR: Electronic medical record

pathway), or nothing, to improve management of patients in a primary care setting (Table 4). Amongst physicians and APPs, telemedicine service availability (i.e., a neurologist available by telephone) was the most preferred intervention (42\%), followed by a care pathway in the EMR (32\%) and monthly physician visits $(26 \%)$. These proportions were not significantly different by chi-square test $(p=0.076)$. Job role also did not predict preference for any individual response $(p=0.19$ 0.54). Notably, no respondents selected "no change", suggesting overall preference for improvement in the current methods in which patients with conditions that may require referral to neurologic specialists are managed in primary care.

\section{Discussion}

Our data reveal that person-to-person communication is most frequently preferred by primary care team members to coordinate patient care with specialty clinics. Several studies have demonstrated improved patient health outcomes using interventions that employed person-to-person communication strategies to bridge the primary care-specialty interface $[15,17-20]$. The studies suggest that personto-person modalities are not only preferred, but also are effective. This is consistent with the desire of physicians and specialists to develop a personal working relationship with one another to coordinate care $[23,24]$. In an ever-changing landscape of healthcare communication options largely driven by digital technology and EMRs, preservation of direct person-to-person communication is of value and has importance.

Additional preferred modalities included EMR chat, a secure pager and fax. Interestingly, physicians were more likely to prefer a secure pager compared to other team members for communicating both direct and indirect patient care matters. This may be due to physicians being more familiar with paging systems, which in the past have been commonly used for on-call responsibilities in both inpatient and outpatient settings. Additionally, the paging system used by MHPP is essentially a secure form of text messaging, allowing providers to communicate detailed messages with one another using their personal cell phones. While direct verbal communication was still most preferred by physicians as a group, our data suggest that physicians may also be amenable to using modern paging systems to coordinate care with one another in an outpatient setting.

The second most preferred modality for coordination of both direct and indirect matters was EMR chat, which resembles a secure form of "instant messaging" between two providers or staff within the patient's medical record. The efficacy of this mechanism has been poorly studied and requires that the primary care and specialty offices utilize a shared EMR. However, one can gain insight from integrated healthcare systems with universal EMRs that employ eConsults, a service whereby primary care providers contact specialists electronically via the EMR for help deciding whether a particular patient requires a face-to-face consultation. One study found that eConsult use reduced unnecessary neurology referrals by $50 \%$ [25]. eConsults are perceived positively by primary care physicians but are more likely to be viewed negatively by specialists, likely because they may not be reimbursed for time spent on the consult $[8,26]$. This issue could be true of any intervention that increases time spent on the part of the provider. Thus, reimbursement systems may need to be revised for meaningful collaboration across specialties to be sustainable. Our exploratory study was commissioned as a quality improvement project by MHPP, and as such did not involve reimbursement of the investigative team or those who were surveyed.

A variety of meta-analyses have examined how increasing communication between primary care and specialty offices modulates patient health outcomes $[21,22,27,28]$, the majority of which report an overall positive effect of collaborative interventions. A recent metaanalysis by Smith et al. demonstrated that shared care interventions led to reduced blood pressure in patients with hypertension, diabetes, and chronic kidney disease, and improved mood in patients with depression [21]. However, the authors found no reduction in hospital admissions or service usage. A major limitation in assessing the efficacy of collaborative care interventions is that most studies utilize a combination of strategies that vary across reports. For example, in some instances the primary care physician may be required to speak to a collaborating specialist by phone at regular intervals, or by contrast, the specialist may simply be on-call for the primary care team to contact on an as-needed basis. Thus, it is difficult to assess what specific aspect of the intervention confers patient benefit. Further, the majority of published work examines a limited number of medical specialties, namely psychiatry and endocrinology.

In addition to elucidating preferred communication methods for patient care matters, our results also demonstrate that primary care physicians and APPs are interested in employing additional strategies such as monthly neurologist visits, a teleneurology service, and neurology-specific EMR order sets to improve management of neurological conditions in the primary care setting. Teleneurology has been used successfully in outpatient settings and found to be safe, wellperceived by patients and clinicians, and leads to similar outcomes when compared to face-to-face visits [29-34]. In most instances, teleneurology has been used in place of face-to-face patient evaluations, especially in rural or underserved populations, but has not been specifically studied as a mechanism to improve communication between primary care physicians and neurologists. In our survey, we envisioned having an on-call neurologist that primary care team members could consult by phone during business hours. The consultant would conduct brief assessments lending themselves to triage of a patient to additional outpatient diagnostic testing or treatment in the primary care setting, referral to the neurology clinic in more complex cases, or in extreme cases to admission to the hospital. This intervention would complement the preference of primary care team members for person-to-person communication, but may require the creation of a new position for an on-call neurologist, as outpatient neurologists may not be amenable to answering calls from primary care providers while also trying to see scheduled patients in a busy neurologic clinic setting.

The second intervention we suggested was generating neurologyspecific order sets or clinical pathways in the EMR to help primary care providers improve diagnostic study or management of neurological conditions. Specialized EMR order sets appear to be beneficial in the evaluation and treatment of acute conditions such as ischemic stroke $[35,36]$, but it is unknown how they alter patient outcomes in outpatient neurology. A similar number of primary care physicians and APPs selected monthly neurologist visits as an opportunity to increase knowledge of neurological conditions and ask questions about 
individual cases. This type of intervention has not been well studied in neurological practice, and data are mixed in other specialties. One report showed that education of primary care physicians alone was insufficient to alter outcomes [37], while another suggested that a combination of primary care physician education and access to psychiatry consultation improved outcomes in depression [16]. Regular specialist visits appear to be more feasible in health systems where primary care and neurology are either co-located or close in geographical proximity but may be especially useful in primary care clinics in underserved populations. Taken together, primary care providers were amenable to a variety of interventions, however, more work is needed to determine how effectively these systems may improve patient care.

In relation to study limitations, we acknowledge that our findings are limited to a single health system, and one cannot conclude that our findings are applicable across a variety of healthcare systems. Further, it will be important to assess whether specialty team members share similar communication preferences, and whether implementation of these preferences leads to positive patient outcomes. Also, our study was completed in 2016, thus technology and preferences for technology may have changed. It would also have been interesting to know if age predicted preference for communication modality, however our database did not include the age of the study participants. We suspect that there may be a generational gap in relation to preference for different types of communication methods.

In sum, our findings provide a framework for implementation of interventions designed to improve the primary care-neurology communication interface. In particular, our data suggest that person-to-person communication, perhaps in combination with asynchronous modalities such as EMR chat and paging systems, may improve collaboration between primary care and neurology offices. Interventions could involve joint consultations or regular meetings between primary care and neurology team members either in a faceto-face or teleneurology context. Finally, interventions should be carefully crafted to minimize the amount of additional workload placed on providers and staff to foster permanent, sustainable collaboration across disciplines.

\section{References}

1. Andel C, Davidow SL, Hollander M, Moreno DA (2012) The economics of health care quality and medical errors. J Health Care Finance 39: 39-50. [Crossref]

2. Makary MA, Daniel M (2016) Medical error-the third leading cause of death in the US. BMJ 353: i2139. [Crossref]

3. David G, Gunnarsson CL, Waters HC, Horblyuk R, Kaplan HS (2013) Economic Measurement of Medical Errors Using a Hospital Claims Database. Value Health 16: 305-310. [Crossref]

4. James JT (2013) A New, Evidence-based Estimate of Patient Harms Associated with Hospital Care. J Patient Saf 9:122-128. [Crossref]

5. Fisher ES (2008) Building a medical neighborhood for the medical home. $N$ Engl $J$ Med 359: 1202-1205. [Crossref]

6. Burwell SM (2015) Setting Value-Based Payment Goals - HHS Efforts to Improve U.S. Health Care. N Engl J Med 372: 897-899. [Crossref]

7. Gorelick PB (2016) Adaptation of neurological practice and policy to a changing US health-care landscape. Lancet Neurol 15: 444-450. [Crossref]

8. Zuchowski JL, Rose DE, Hamilton AB, Stockdale SE, Meredith LS, et al. (2015) Challenges in referral communication between VHA primary care and specialty care. $J$ Gen Intern Med 30: 305-311. [Crossref]

9. Travaline JM, Ruchinskas R, D'Alonzo GE Jr (2005) Patient-Physician communication: why and how. J Am Osteopath Assoc 105: 13-18. [Crossref]
10. Altini E, Martignoni, Lablanca R (2014) Communication models for doctor-patient relationships. J Cancer Educ 29: 211-212. [Crossref]

11. Kurtz SM. Doctor-patient communication: principles and practices. Can J Neurol Sci 29: S23-S29. [Crossref]

12. Ha JF, Longnecker N (2010) Doctor-patient communication: a review. Ochsner $J 10$ : 38-43. [Crossref]

13. Center for Disease Control and Prevention. National Ambulatory Medical Care Survey: 2015 State and National Summary Tables. Available from: https://www.cdc.gov/nchs/ data/ahcd/namcs_summary/2015_namcs_web_tables.pdf. Accessed Jan. 25, 2018.

14. Association of American Medical Colleges. 2018 Physician Specialty Data Report. Available from: https://www.aamc.org/data/workforce/reports/492536/2018physician-specialty-data-report.html. Accessed Jan. 25, 2018.

15. Katzelnick DJ, Simon GE, Pearson SD, Manning WG, Helstad CP, et al. (2000) Randomized Trial of a Depression Management Program in High Utilizers of Medical Care. Arch Fam Med 9: 345-351. [Crossref]

16. Worrall G, Angel J, Chaulk P, Clarke C, Robbins M (1999) Effectiveness of an educational strategy to improve family physicians' detection and management of depression: a randomized controlled trial. CMAJ 161: 37-40. [Crossref]

17. Katon W, Russo J, Von Korff M, Lin E, Simon G, et al. (2002) Long-term effects of a collaborative care intervention in persistently depressed primary care patients. $J$ Gen Intern Med 17: 741-748. [Crossref]

18. Hilty DM, Marks S, Wegelin J, Callahan EJ, Nesbitt TS (2007) A randomized, controlled trial of disease management modules, including telepsychiatric care, for depression in rural primary care. Psychiatry (Edgmont) 4: 58-65. [Crossref]

19. Dietrich AJ, Oxman TE, Burns MR, Winchell CW, Chin T (2003) Application of a Depression Management Office System in Community Practice: A Demonstration. $J$ Am Board Fam Pract 16: 107-114. [Crossref]

20. Muntingh A, van der Feltz-Cornelis C, van Marwijk H, Spinhoven P, Assendelft W, et al. (2014) Effectiveness of Collaborative Stepped Care for Anxiety Disorders in Primary Care: A Pragmatic Cluster Randomised Controlled Trial. Psychother Psychosom 83: 37-44. [Crossref]

21. Smith S, Cousins G, Clyne B, Allwright S, O’Dowd T (2017) Shared care across the interface between primary and specialty care in management of long term conditions. Cochrane Database Syst Rev 2: CD004910. [Crossref]

22. Foy R, Hempel S, Rubenstein L, Suttorp M, Seelig M, et al. (2010) Meta-analysis: effect of interactive communication between collaborating primary care physicians and specialists. Ann Intern Med 152: 247-258. [Crossref]

23. Berendsen AJ, Benneker W, Jong B, Klazinga NS, Schuling J (2007) Motives and preferences of general practitioners for new collaboration models with medical specialists: a qualitative study. BMC Health Serv Res 7: 4. [Crossref]

24. Berendsen AJ, Benneker W, Schuling J, Rijkers-Koorn N, Slaets JP, et al. (2006) Collaboration with general practitioners: preferences of medical specialists - a qualitative study. BMC Health Serv Res 6: 155. [Crossref]

25. Bradi AC, Sitwell L, Liddy C, Afkham A, Keely E (2018) Ask a neurologist: What primary care providers ask, and reducing referrals through eConsults. Neurol Clin Pract 8: 186-191. [Crossref]

26. Vimalananda VG, Gupte G, Seraj SM, Orlander J, Berlowitz D, et al. (2015) Electronic consultations (e-consults) to improve access to specialty care: A systematic review and narrative synthesis. J Telemed Telecare 21: 323-330. [Crossref]

27. Bower P, Gilbody S, Richards D, Fletcher J, Sutton A (2006) Collaborative care for depression in primary care: Making sense of a complex intervention: systematic review and meta-regression. Br J Psychiatry 189: 484-493. [Crossref]

28. Gunn J, Diggens J, Hegarty K, Blashki G (2006) A systematic review of complex system interventions designed to increase recovery from depression in primary care. BMC Health Serv Res 6: 88. [Crossref]

29. Müller KI, Alstadhaug KB, Bekkelund SI (2017) A randomized trial of telemedicine efficacy and safety for nonacute headaches. Neurology 89: 153-162. [Crossref]

30. Davis LE, Ann H, Anne L, Pirio R, Amanda F, et al. (2019) Using Teleneurology to Deliver Chronic Neurologic Care to Rural Veterans: Analysis of the First 1,100 Patient Visits. Telemed J E Health 25: 274-278. [Crossref]

31. Wechsler LR (2015) Advantages and limitations of teleneurology. JAMA Neurol 72 349-354. [Crossref] 
32. Dorsey RE, Deuel LM, Voss TS, Finnigan K, George BP, et al. (2010) Increasing access to specialty care: A pilot, randomized controlled trial of telemedicine for Parkinson's disease. Mov Disord 25: 1652-1659. [Crossref]

33. Kane RL, Bever CT, Ehrmantraut M, Forte A, Culpepper WJ, et al. (2008) Teleneurology in patients with multiple sclerosis: EDSS ratings derived remotely and from hands-on examination. J Telemed Telecare 14: 190-194. [Crossref]

34. Ahmed S, Mann C, Sinclair BD, Heino A, Iskiw B, et al. (2008) Feasibility of epilepsy follow-up care through telemedicine: A pilot study on the patient's perspective. Epilepsia 49:573-585. [Crossref]
35. Ballard DW, Kim AS, Huang J, Park DK, Kene MV, et al. (2015) Implementation of Computerized Physician Order Entry Is Associated with Increased Thrombolytic Administration for Emergency Department Patients With Acute Ischemic Stroke. Ann Emerg Med 66: 601-610. [Crossref]

36. Yang JM, Park YS, Chung SP, Chung HS, Lee HS et al. (2014) Implementation of a clinical pathway based on a computerized physician order entry system for ischemic stroke attenuates off-hour and weekend effects in the ED. Am J Emerg Med 32: 884-889. [Crossref]

37. Christensen H, Griffiths KM, Gulliver A, Clack D, Kljakovic M, et al. (2008) Models in the delivery of depression care: A systematic review of randomised and controlled intervention trials. BMC Fam Pract 9: 25. [Crossref]

Copyright: $(2020$ Woodworth HL. This is an open-access article distributed under the terms of the Creative Commons Attribution License, which permits unrestricted use, distribution, and reproduction in any medium, provided the original author and source are credited. 